\title{
Das Weißbuch Digitale Plattformen
}

Nach dem Grünbuch Digitale Plattformen hat das Bundesministerium für Wirtschaft und Energie (BMWi) nun auch das entsprechende Weißbuch „Digitale Plattformen: Digitale Ordnungspolitik für Wachstum, Innovation, Wettbewerb und Teilhabe" herausgebracht. Mit dem Weißbuch verfolgt das BMWi nach eigener Aussage vier Ziele, die bei der "Gestaltung der Digitalisierung" erreicht werden sollen: Erstens soll ein politisch begleiteter Prozess der Digitalisierung stattfinden. Zweitens soll die Wachstumsdynamik positiv vom Staat beeinflusst werden. Drittens soll durch die Errichtung geeigneter Institutionen für Sicherheit gesorgt werden. Und viertens soll auch auf europäischer Ebene ein einheitlicher Rechtsrahmen entstehen.

Insbesondere das erste Ziel ist aus wirtschaftspolitischer Sicht interessant. Tatsächlich ist es wichtig, sich der Digitalisierung und den damit verbundenen Herausforderungen anzunehmen, und Anpassungen des Rechtsrahmens und der Wirtschaftspolitik - wenn nötig - durchzuführen. Dazu ist es erforderlich, dass die komplexen ökonomischen Effekte hinter der sogenannten Plattformökonomie genauer analysiert werden. Ein Beispiel für eine notwendige Anpassung enthält etwa die anstehende neunte Novelle des Gesetzes gegen Wettbewerbsbeschränkungen. Darin ist festgelegt, dass die Unentgeltlichkeit einer Leistung der Annahme nicht entgegenstehe, dass es sich hier um einen Markt handelt. Damit wird z.B. sichergestellt, dass in Zukunft auch Inhaltemärkte bei Nachrichtenplattformen und Zuschauermärkte im FreeTV nicht nur ökonomisch, sondern auch juristisch als Markt verstanden werden.

Wie aber wird der Prozess der Digitalisierung darüber hinaus in Zukunft begleitet? Im Weißbuch wird dazu ausgeführt, dass statt einer „Disruption“ eine "gestaltete Transformation der Plattformökonomie“ angestrebt werde. „Klare Regeln" sollen diesen Prozess ermöglichen, eine „ungesteuerte Digitalisierung“ solle dagegen nicht stattfinden. Auch werden vor allem die großen „Plattformen als Platzhirsche der Digitalwirtschaft" beklagt, die „dank Big Data den zentralen Kundenzugang organisieren und orchestrieren“ sowie "Konsumenten- und etliche Dienstleistungsmärkte quasi überrollt" hätten. Es wird zwar ebenso eingeräumt, dass die Plattformen die Treiber der digitalen Entwicklung seien, grundsätzlich lässt sich aber aus dem Weißbuch eine gewisse Grundskepsis gegenüber den Unternehmen der digitalen Ökonomie herauslesen. Auch wird beklagt, dass die Internetplattformen die analogen Unternehmen verdrängten.

Das Weißbuch verkennt bei dem Ziel der gestalteten Transformation jedoch, dass insbesondere drastische Innovationen nicht selten Disruption (oder wie Schumpeter es einmal ausgedrückt hat: schöpferische Zerstörung) mit sich bringen. Neue, überlegene Technologien und Geschäftsmodelle verdrängen zwangsläufig die ineffizienteren. Will man dagegen, wie das Weißbuch postuliert, keine Disruption und schützt man veraltete Modelle staatlich gegen die effizienteren, verzichtet man auf wirtschaftliche und gesellschaftliche Vorteile der Digitalisierung. Vor allem aber riskiert man, im Digitalsektor von anderen Ländern abgehängt zu werden und auf Dauer hinter diesen zurückzubleiben.

Ein wichtiges Thema, das vom Weißbuch in diesem Zusammenhang aufgegriffen wird, ist die Relevanz von Big Data. Völlig zu Recht wird darauf verwiesen, dass es kein wirtschaftspolitisches Ziel sein kann, Datenerhebung und -verarbeitung übermäßig einzuschränken oder gar zu unterbinden. Dies gilt insbesondere auch deshalb,

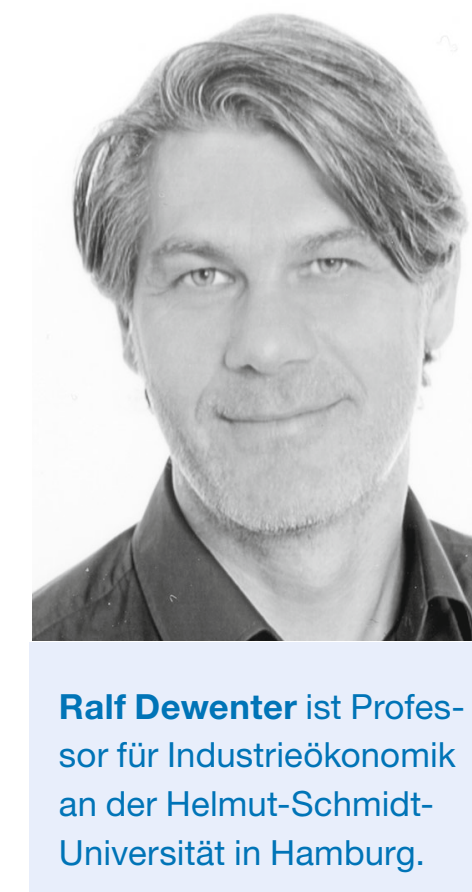

Ralf Dewenter ist Professor für Industrieökonomik an der Helmut-SchmidtUniversität in Hamburg. 
weil die enormen Vorteile der Datenerhebung und -analyse weit über die Anwendung durch digitale Plattformen hinausgehen. Im gleichen Zusammenhang wird jedoch auf die Gefahr von „Datenmonopolen“ und einer „stärkeren Ausschließlichkeit“ verwiesen. Es besteht also anscheinend die diffuse Befürchtung, dass wenige digitale Plattformen alle relevanten Daten, die durch die Digitalisierung entstehen, ausschließlich für sich behalten könnten. Dies verkennt jedoch die wesentlichen Charakteristika von Daten: Denn diese sind zum einen zwar ausschließbar. Zum anderen jedoch, und dies sind weitere wichtige Eigenschaften, sind Daten in der Regel weder rival noch exklusiv. So kann jeder Nutzer darüber entscheiden, ob er seine Daten einer Plattform zur Verfügung stellt oder nicht. Gleichzeitig verschlechtern sich diese Daten jedoch nicht, wenn diese Informationen mehreren Plattformen überlassen werden. Plattformen können also oftmals gar nicht verhindern, dass dieselben Daten von mehreren Konkurrenten gleichzeitig genutzt werden. Datenmonopole sind im Bereich von Big Data also wenig wahrscheinlich.

Eng verbunden mit der Frage der Relevanz von Big Data ist das Thema der Eigentumsund Nutzungsrechte an Daten. In diesem Zusammenhang diskutiert das Weißbuch unter anderem die Schaffung neuer Ausschließlichkeitsrechte und die Stärkung von Zugangsrechten für Daten. Während das Datenschutzrecht personenbezogene Daten gegen Missbrauch schützt, sind Daten, die nicht personenbezogen sind und z.B. durch Maschinen erzeugt werden, in der Regel durch Rechte geistigen Eigentums geschützt. Aus ökonomischer Sicht ist es zunächst wichtig, dass Nutzungsrechte übertragen werden und somit Marktlösungen umgesetzt werden können. Erzwungene Datenzugangsrechte würden bestehende Rechte aushebeln und damit Gefahr laufen, ein Trittbrettfahrerverhalten zu erzeugen und damit die Anreize zur Erhebung von Daten massiv zu reduzieren. Auch die Schaffung neuer Ausschließlichkeitsrechte erscheint bislang nicht notwendig. Vielmehr sollte die im Weißbuch angesprochene dritte Option, die der vertraglichen Lösungen, verstärkt verfolgt werden.

Tatsächlich besteht Big Data jedoch aus mehr als nur der Sammlung von Daten. Unterschätzt wird regelmäßig die damit verbundene Fähigkeit, Daten effizient auswerten zu können. Viel wichtiger als die Möglichkeit, Daten zu erheben, sind das zugrundeliegende Know-how und die dazu notwendige Technologie. Selbstlernende Algorithmen, sogenanntes Deep Learning und Artificial Intelligence spielen dabei eine wesentliche Rolle. Allerdings geht es dabei nicht so sehr darum, wie vom Weißbuch proklamiert, dass Nutzer die Funktionsweise der Algorithmen verstehen oder das Zustandekommen von Rankings transparent wird. Viel wichtiger ist es, die Vorteile dieser Algorithmen zu nutzen. So soll maschinelles Lernen helfen, die Präferenzen der Nutzer möglichst gut abzubilden und den Wettbewerb zu stärken. Verhindert werden sollte dagegen, dass etwa selbstlernende Algorithmen den Wettbewerb in irgendeiner Form beschränken.

Die wirtschaftspolitischen Herausforderungen der Digitalisierung sind vielfältig und die Diskussionen und Analysen, die mit Grün- und Weißbuch zu diesem Thema angestoßen wurden, sind wichtige Voraussetzungen für notwendige Anpassungen des Ordnungsrahmens. Dabei sollte jedoch darauf geachtet werden, dass auch weiterhin eine so dynamische Entwicklung von Unternehmen, deren Geschäftsmodelle die Vorteile der Digitalisierung nutzen, möglich sein wird. Dabei darf zum einen der Konsument nicht aus den Augen verloren werden, zum anderen müssen die notwendigen Anpassungen ökonomisch fundiert sein. Gerade beim Umgang mit digitalen Plattformen und Big Data sollte daher nicht voreilig gehandelt werden. Datenmonopole, Ausschließlichkeitsrechte für Daten oder auch scheinbar monopolistische Plattformen sind dabei vor allem in einem dynamischen Wettbewerbsumfeld ein viel geringeres Problem, als es auf den ersten Blick erscheint. 